\title{
A NONLINEAR THEOREM OF ERGODIC TYPE.II
}

\author{
G. FEATHERS AND W. G. DOTSON, JR.
}

\begin{abstract}
A theorem is proved concerning the relationship between a certain collection of mappings and a fixed point for that collection of mappings. The conditions on the mappings are very similar to ones in a paper by W. F. Eberlein.
\end{abstract}

THEOREM. Let $B$ be a uniformly convex Banach space with a weak duality mapping [2] and $C$ a closed convex subset of B. Suppose $G=\left\{S_{m}\right\}_{m=1}^{\infty}$ is a collection of self-mappings of $C$ such that $I-S_{m}$ is demiclosed $\forall S_{m} \in G$. Suppose further that there is a collection of nonexpansive self-mappings of $C$, $\left\{C_{n}\right\}_{n=1}^{\infty}$ satisfying

(a) $C_{n}(x) \in\left\{S_{m}(x): S_{m} \in G\right\}^{-}$for all $x \in C$,

(b) there exist $x_{0}$ such that

$$
\begin{aligned}
& C_{n} S_{m}\left(x_{0}\right)-C_{n}\left(x_{0}\right) \rightarrow 0 \quad \text { as } n \rightarrow \infty, \\
& S_{m} C_{n}\left(x_{0}\right)-C_{n}\left(x_{0}\right) \rightarrow 0 \quad \text { as } n \rightarrow \infty,
\end{aligned}
$$

(c) suppose there exists a subsequence $\left\{C_{n_{j}}\left(x_{0}\right)\right\}$ of $\left\{C_{n}\left(x_{0}\right)\right\}_{n=1}^{\infty}$ such that

$$
C_{n}\left(x_{0}\right) \rightarrow y \in C \quad[\text { since } C \text { is weakly closed }]
$$

then

(1) $S_{m}(y)=y$ for all $S_{m} \in G$,

(2) $C_{n}\left(x_{0}\right) \rightarrow y$.

LEMMA 1. Under the above conditions the following is true: $C_{n}\left(S\left(x_{0}\right)\right)-$ $C_{n}\left(x_{0}\right) \rightarrow 0$ as $n \rightarrow \infty$ where $S\left(x_{0}\right)$ is any element of $\left\{S_{m}\left(x_{0}\right): S_{m} \in G\right\}^{-}$.

Proof. Clearly we need only check limit points of $\left\{S_{m}\left(x_{0}\right): S_{m} \in G\right\}$. So let $S\left(x_{0}\right)$ be a limit point of $\left\{S_{m}\left(x_{0}\right): S_{m} \in G\right\}$, and let $S_{m_{j}}\left(x_{0}\right) \rightarrow S\left(x_{0}\right)$. Given $\varepsilon>0$ there exists $m_{J_{1}}$ such that $\left\|S_{m_{l}}\left(x_{0}\right)-S\left(x_{0}\right)\right\|<\varepsilon / 2$ for all $m_{J} \geqslant m_{J_{1}}$. Now consider

$$
\begin{aligned}
& \left\|C_{n}\left(S\left(x_{0}\right)\right)-C_{n}\left(x_{0}\right)\right\| \\
& \quad=\left\|C_{n}\left(S\left(x_{0}\right)\right)-C_{n}\left(S_{m_{J}}\left(x_{0}\right)\right)+C_{n}\left(S_{m_{J}}\left(x_{0}\right)\right)-C_{n}\left(x_{0}\right)\right\| \\
& \quad \leqslant\left\|C_{n}\left(S\left(x_{0}\right)\right)-C_{n}\left(S_{m_{J}}\left(x_{0}\right)\right)\right\|+\left\|C_{n} S_{m_{J}}\left(x_{0}\right)-C_{n}\left(x_{0}\right)\right\|
\end{aligned}
$$

Received by the editors September 26, 1977.

AMS (MOS) subject classifications (1970). Primary 47H10; Secondary 47H99. 
which if we pick $m_{J_{2}}>m_{J_{1}}$ we have that

$$
\begin{aligned}
& \left\|C_{n}\left(S\left(x_{0}\right)\right)-C_{n}\left(x_{0}\right)\right\| \\
& \leqslant\left\|C_{n}\left(S\left(x_{0}\right)\right)-C_{n}\left(S_{m_{J_{2}}}\left(x_{0}\right)\right)\right\|+\left\|C_{n}\left(S_{m_{J_{2}}}\left(x_{0}\right)\right)-C_{n}\left(x_{0}\right)\right\| \\
& \quad-\left\|S\left(x_{0}\right)-S_{m_{J_{2}}}\left(x_{0}\right)\right\|+\left\|C_{n}\left(S_{m_{J_{2}}}\left(x_{0}\right)\right)-C_{n}\left(x_{0}\right)\right\|
\end{aligned}
$$

[by nonexpansiveness of the $C_{n}$ 's] $\leqslant \varepsilon / 2+\left\|C_{n}\left(S_{m_{J}}\left(x_{0}\right)\right)-C_{n}\left(x_{0}\right)\right\|$ for which there exists $n_{0}$ such that for all $n \geqslant n_{0} \leqslant \varepsilon / 2+\varepsilon / 2$ (by condition (b)). So for all $n \geqslant n_{0}$ we have $\left\|C_{n}\left(S\left(x_{0}\right)\right)-C_{n}\left(x_{0}\right)\right\|<\varepsilon \Rightarrow \| C_{n}\left(S\left(x_{0}\right)\right)-$ $C_{n}\left(x_{0}\right) \| \rightarrow 0$ as $n \rightarrow \infty$.

We will now show $S_{m}(y)=y$ for all $S_{m} \in G$. We have that $C_{n_{j}}\left(x_{0}\right) \rightarrow y$, from now on $C_{n_{j}}$ will be reindexed by $L$, i.e. $C_{L}\left(x_{0}\right) \rightarrow y$. By condition (c) $\left(I-S_{m}\right) C_{n}\left(x_{0}\right) \rightarrow 0 \Rightarrow\left(I-S_{m}\right) C_{L}\left(x_{0}\right) \rightarrow 0$ so by the demiclosedness of $I-$ $S_{m}$ (for all $S_{m} \in G$ ) we have $\left(I-S_{m}\right) y=0$ or $y=S_{m}(y)$ for all $S_{m} \in G$. Also since $C_{n}(y) \in\left\{S_{m}(y): S_{m} \in G\right\}^{-}=\{y\}^{-}=\{y\}$ we have $C_{n}(y)=y$ for all $n$.

The set $\left\{C_{n}\left(x_{0}\right)\right\}$ is a bounded set because $\left\|C_{n}\left(x_{0}\right)-y\right\|=\| C_{n}\left(x_{0}\right)-$ $C_{n}(y)\|\leqslant\| x_{0}-y\|\Rightarrow\| C_{n}\left(x_{0}\right)\|\leqslant\| x_{0}-y\|+\| y \|$ for all $n$. Consider the subset $\left\{C_{n}\left(x_{0}\right)\right\} \backslash\left\{C_{L}\left(x_{0}\right)\right\} \subseteq\left\{C_{n}\left(x_{0}\right)\right\}$ this is clearly bounded which implies, since uniformly convex Banach spaces are reflexive, that there exists a weakly convergent subsequence, call it $C_{s}\left(x_{0}\right) \rightarrow y_{1}$. We will show $y=y_{1}$.

Now by nonexpansiveness of the $C_{n}$ 's we have $\left\|C_{L}\left(C_{s}\left(x_{0}\right)\right)-y\right\|=$ $\left\|C_{L}\left(C_{s}\left(x_{0}\right)\right)-C_{L}(y)\right\| \leqslant\left\|C_{s}\left(x_{0}\right)-y\right\|$.

We know that $C_{L}\left(C_{s}\left(x_{0}\right)\right)-C_{L}\left(x_{0}\right) \rightarrow 0$ as $L \rightarrow \infty$ by assumption (2), Lemma 1, and the fact that $C_{s}\left(x_{0}\right) \in\left\{S_{m}\left(x_{0}\right): S_{m} \in G\right\}^{-}$. So there exists $L_{0}$ such that for all $L \geqslant L_{0}\left\|C_{L}\left(C_{s}\left(x_{0}\right)\right)-C_{L}\left(x_{0}\right)\right\|<\varepsilon$, given $\varepsilon<0$. Now $\left|\left\|C_{L} C_{s}\left(x_{0}\right)-y\right\|-\left\|C_{L}\left(x_{0}\right)-y\right\|\right| \leqslant\left\|C_{L}\left(C_{s}\left(x_{0}\right)\right)-C_{L}\left(x_{0}\right)\right\|<\varepsilon$ for all $L \geqslant L_{0} \Rightarrow$ that $\left\|C_{L}\left(x_{0}\right)-y\right\| \leqslant\left\|C_{L}\left(C_{s}\left(x_{0}\right)\right)-y\right\|+\varepsilon \leqslant\left\|C_{s}\left(x_{0}\right)-y\right\|+\varepsilon$ for all $L \geqslant L \Rightarrow \operatorname{Lim}\left\{\left\|C_{L}\left(x_{0}\right)-y\right\|\right\} \leqslant\left\|C_{s}\left(x_{0}\right)-y\right\|$. This holds for all $C_{s}\left(x_{0}\right) \Rightarrow \operatorname{Lim}\left\{\left\|C_{L} \overline{\left(x_{0}\right)}-y\right\|\right\} \leqslant \operatorname{Lim}\left\{\left\|C_{S}\left(x_{0}\right)-y\right\|\right\}$. Now by interchanging the roles of $C_{L}$ and $C_{S}$ we get $\underline{\operatorname{Lim}}\left\{\left\|C_{S}\left(x_{0}\right)-y\right\|\right\} \leqslant \underline{\operatorname{Lim}}\left\{\left\|C_{L}\left(x_{0}\right)-y\right\|\right\}$ which $\Rightarrow$

$$
\underline{\operatorname{Lim}}\left\{\left\|C_{S}\left(x_{0}\right)-y\right\|\right\}=\underline{\operatorname{Lim}}\left\{\left\|C_{L}\left(x_{0}\right)-y\right\|\right\} .
$$

Let $d=\operatorname{Lim}\left\{\left\|C_{S}\left(x_{0}\right)-y\right\|\right\}$ then extract a subsequence such that $d=$ $\operatorname{Lim}\left\|C_{T}\left(x_{0}\right)-y\right\|$, and $d=\operatorname{Lim}\left\|C_{U}\left(x_{0}\right)-y\right\|$ where $C_{T}\left(x_{0}\right)$ is a subsequence of $C_{S}\left(x_{0}\right)$ and $C_{U}\left(x_{0}\right)$ is a subsequence of $C_{L}\left(x_{0}\right)$. Now replace in the previous argument $C_{L}\left(x_{0}\right)$ by $C_{U}\left(x_{0}\right), C_{S}\left(x_{0}\right)$ by $C_{T}\left(x_{0}\right)$ and $y$ by $y_{1}$ [using the fact that since $C_{S}\left(x_{0}\right) \rightarrow y_{1} \Rightarrow C_{n}\left(y_{1}\right)=y_{1}$, same proof as before with $y$ replaced by $\left.y_{1}\right]$. The result of this is that $\underline{\operatorname{Lim}}\left\{\left\|C_{U}\left(x_{0}\right)-y_{1}\right\|\right\}=\underline{\operatorname{Lim}}\left\{\left\|C_{T}\left(x_{0}\right)-y_{1}\right\|\right\}$, so as before extract subsequences such that

$$
d_{1}=\underline{\operatorname{Lim}}\left\{\left\|C_{U}\left(x_{0}\right)-y_{1}\right\|\right\}=\operatorname{Lim}\left\|C_{V}\left(x_{0}\right)-y_{1}\right\|=\operatorname{Lim}\left\|C_{W}\left(x_{0}\right)-y_{1}\right\|
$$

where $C_{V}\left(x_{0}\right)$ is a subsequence of $C_{U}\left(x_{0}\right)$ and $C_{W}\left(x_{0}\right)$ is a subsequence of 
$C_{T}\left(x_{0}\right)$. We now have that

$$
\begin{gathered}
d=\operatorname{Lim}\left\|C_{V}\left(x_{0}\right)-y\right\|=\operatorname{Lim}\left\|C_{W}\left(x_{0}\right)-y\right\|, \\
d_{1}=\operatorname{Lim}\left\|C_{V}\left(x_{0}\right)-y_{1}\right\|=\operatorname{Lim}\left\|C_{W}\left(x_{0}\right)-y_{1}\right\| \text { and } \\
C_{V}\left(x_{0}\right) \rightarrow y, \quad C_{W}\left(x_{0}\right)-y_{1} .
\end{gathered}
$$

Now using Lemma 3 of [2] we have that, since $C_{V}\left(x_{0}\right) \rightarrow y, \operatorname{Lim} \| C_{V}\left(x_{0}\right)-$ $y\|\leqslant \operatorname{Lim}\| C_{V}\left(x_{0}\right)-y_{1} \|$ i.e. $d \leqslant d_{1}$, but since $C_{W}\left(x_{0}\right) \rightarrow y_{1}$ this implies $\operatorname{Lim}\left\|C_{W}\left(x_{0}\right)-y_{1}\right\| \leqslant \operatorname{Lim}\left\|C_{W}\left(x_{0}\right)-y\right\|$ i.e. $d \leqslant d_{1}$ so that $d=d_{1}$, which by Lemma $3 y=y_{1}$.

REMARK 1. This is a generalization of Opial's Theorem 2 in [2], with $S_{m}=T^{m}$ and $C_{n}=T^{n}$. Opial in [2] showed that if $T$ was nonexpansive then $I-T^{m}$ was demiclosed in a uniformly convex Banach space with a weakly continuous duality mapping, so $\left\{S_{m}\right\}$ satisfying the conditions imposed on them. Also condition (1) is trivially satisfied, and the asymptotic regularity of $\Gamma$ implies condition (2). Since $T$ has a fixed point by assumption this implies there exists a weakly convergent subsequence, for each $x \in C_{1}$ of $\left\{C_{n}(x)\right\}_{n=1}^{\infty}$, i.e. $C_{n_{s}}(x) \rightarrow y_{x}$. So our theorem says that

(1) $T^{n}\left(y_{x}\right)=y_{x} \forall n$ and

(2) $C_{n}(x) \rightarrow y_{x}$.

Also since Opial's Theorem 2 implies Theorem 3 our theorem implies Theorem 3, which is a partial affirmative answer to $\mathrm{H}$. Schaefer's conjecture. Unfortunately it adds no new information on the conjecture.

REMARK 2. The conditions of this theorem are very similar to the ones of the mean ergodic theorem of Eberlein [1].

\section{REFERENCES}

1. W. F. Eberlein, Abstract ergodic theorems and weak almost periodic functions, Trans. Amer. Math. Soc. 67 (1949), 217-240.

2. Z. Opial, Weak convergence of the sequence of successive approximations for nonexpansive mappings, Bull. Amer. Math. Soc. 73 (1967), 591-597. MR 35 \#2183.

Department of Mathematics, North Carolina State University at Raleigh, Raleigh, NORTH CAROLINA 27607 